\title{
Do reporting guidelines have an impact? Empirical assessment of changes in reporting before and after the PRISMA extension statement for network meta-analysis
}

\author{
Areti Angeliki Veroniki ${ }^{1,2^{*}}$ (D), Sofia Tsokani ${ }^{1}$, Stella Zevgiti , Irene Pagkalidou ${ }^{3}$, Katerina-Maria Kontouli' \\ Pinar Ambarcioglu ${ }^{4}$, Nikos Pandis $^{5}$, Carole Lunny ${ }^{6}$, Adriani Nikolakopoulou ${ }^{7,8}$, Theodoros Papakonstantinou $^{8}$, \\ Anna Chaimani ${ }^{9,10}$, Sharon E. Straus ${ }^{2,11}$, Brian Hutton ${ }^{12,13}$, Andrea C. Tricco ${ }^{2,14}$, Dimitris Mavridis ${ }^{1,15}$ and \\ Georgia Salanti ${ }^{8}$
}

\begin{abstract}
Background: The Preferred Reporting Items for Systematic Reviews and Meta-Analyses (PRISMA) extension statement for network meta-analysis (NMA) published in 2015 promotes comprehensive reporting in published systematic reviews with NMA. PRISMA-NMA includes 32 items: 27 core items as indicated in the 2009 PRISMA Statement and five items specific to the reporting of NMAs. Although NMA reporting is improving, it is unclear whether PRISMA-NMA has accelerated this improvement. We aimed to investigate the impact of PRISMA-NMA and highlight key items that require attention and improvement.

Methods: We updated our previous collection of NMAs with articles published between April 2015 and July 2018. We assessed the completeness of reporting for each NMA, including main manuscript and online supplements, using the PRISMA-NMA checklist. The PRISMA-NMA checklist originally includes 32 total items (i.e. a 32-point scale original PRISMA-NMA score). We also prepared a modified version of the PRISMA-NMA checklist with 49 items to evaluate separately at a more granular level all multiple-content items (i.e. a 49-point scale modified PRISMA-NMA score). We compared average reporting scores of articles published until and after 2015.

Results: In the 1144 included NMAs the mean modified PRISMA-NMA score was 32.1 (95\% Cl 31.8-32.4) of a possible 49-excellence-score. For 1-year increase, the mean modified score increased by 0.96 ( $95 \% \mathrm{Cl} 0.32$ to 1.59 ) for 389 NMAs published until 2015 and by 0.53 (95\% Cl 0.02 to 1.04) for 755 NMAs published after 2015. The mean modified PRISMA-NMA score for NMAs published after 2015 was higher by 0.81 ( $95 \% \mathrm{Cl} 0.23$ to 1.39) compared to before 2015 when adjusting for journal impact factor, type of review, funding, and treatment category. Description of summary effect sizes to be used, presentation of individual study data, sources of funding for the systematic review, and role of funders dropped in frequency after 2015 by $6-16 \%$.
\end{abstract}

*Correspondence: averoniki@uoi.gr

${ }^{1}$ Department of Primary Education, School of Education, University

of loannina, loannina, Greece

Full list of author information is available at the end of the article give appropriate credit to the original author(s) and the source, provide a link to the Creative Commons licence, and indicate if changes were made. The images or other third party material in this article are included in the article's Creative Commons licence, unless indicated otherwise in a credit line to the material. If material is not included in the article's Creative Commons licence and your intended use is not permitted by statutory regulation or exceeds the permitted use, you will need to obtain permission directly from the copyright holder. To view a copy of this licence, visit http://creativecommons.org/licenses/by/4.0/. The Creative Commons Public Domain Dedication waiver (http://creativecommons.org/publicdomain/zero/1.0/) applies to the data made available in this article, unless otherwise stated in a credit line to the data. 
Conclusions: NMAs published after 2015 more frequently reported the five items associated with NMA compared to those published until 2015. However, improvement in reporting after 2015 is compatible with that observed on a yearly basis until 2015, and hence, it could not be attributed solely to the publication of the PRISMA-NMA.

Keywords: Multiple treatment meta-analysis, PRISMA-NMA, Systematic review, Reporting

\section{Background}

The Preferred Reporting Items for Systematic Reviews and Meta-Analyses (PRISMA) statement was developed to promote comprehensive reporting in published systematic reviews with narrative summary and pairwise meta-analysis to increase transparency and reproducibility [1]. The PRISMA statement, published in 2009, was initially designed for systematic reviews and pairwise meta-analyses of healthcare interventions and has been widely used by reviewers and journals [2]. The statement was updated to PRISMA 2020 to reflect recent advances in the methods of systematic reviews [3]. It is of critical importance to report sufficient and accessible information so that research can be reproduced, which can help avoiding biased recommendations and distort healthcare decision making $[4,5]$.

The PRISMA extension for network meta-analysis (NMA), published in 2015, was prompted by empirical research showing that reporting of NMA was problematic [6]. Our previous scoping reviews including 456 NMAs published until 2015 showed that only a quarter of NMAs were of high methodological quality and that half of the NMAs had failed to report the prerequisite assumptions of evidence synthesis, although both reporting and methodology were found to be improving over time [7, 8]. The PRISMA extension provides guidance for reporting of systematic reviews with NMA and highlights their key reporting components, aiming to improve primarily reporting and indirectly the conduct of reviews with NMA. There are five additional items in the PRISMA extension to NMA: description of methods used to explore network geometry, description of methods used to assess inconsistency, presentation of network diagram, brief overview of network characteristics, and description of results from investigations of inconsistency.

There are few empirical studies that have evaluated the completeness of reporting of NMAs since the publication of the PRISMA extension. The assessment of reporting of 21 systematic reviews with NMAs published until 2017 using the PRISMA-NMA checklist showed that reporting was low in the dental care field [9]. Tonin et al. [10] assessed the extend of compliance with PRISMA (for NMAs published before 2015) and PRISMANMA (for NMAs published between 2015 and end of 2016) in 477 NMAs of pharmacological treatments and showed minor improvement in reporting according to the PRISMA score. A scoping review of 89 NMAs with complementary and alternative medicines published up until 2018 showed that the PRISMA-NMA guideline was overall adequately adopted through key reporting items such as the existence of a protocol, exploring network geometry, and risk of bias assessment were often missing (up to 65\%) [11].

As previous empirical research has suggested that reporting of NMAs is improving over time, it is unclear whether the PRISMA-NMA statement has accelerated this improvement. Our objective was to empirically assess whether the PRISMA-NMA statement had an important impact on the completeness of reporting by comparing NMA articles of randomised controlled trials (RCTs) between two time periods (2013-2015 and 2016-2018). We also aim to investigate publication features (such as journal characteristics or the existence of a protocol) and network characteristics (such as type of interventions compared) that might modify the completeness of reporting. We additionally sought to highlight key items that require further attention and potential improvement moving forward.

\section{Methods}

\section{Eligibility criteria and study selection}

We updated our previous collection of NMAs with articles published between April 2015 and July 2018 using the same search strategy and inclusion criteria as described in our previously published reviews [7, 8, 12]. In brief, networks were eligible if they included RCTs only, included at least four treatment nodes in the network, they had conducted any form of valid indirect comparison or NMA, and the number of studies was larger than the number of treatments compared (see also Appendix 1). In the present study, we included articles published between 2013 and 2018, to have an equal chronological timeframe before and after the PRISMANMA publication (published in June 2015).

\section{Data abstraction}

We developed a predefined data abstraction form in REDCap [13]. We included first author's name, publication year, journal name, and country of corresponding author in the abstracted data. We denoted each journal's impact factor as indicated in the Web of Science (year 
2019). Impact factors for journals not included in this list were obtained from the relevant journals' official website. If an impact factor was not available for year 2019, it was retrieved from a previous year. We grouped NMAs according to the type of treatment comparisons that were presented (pharmacological vs placebo, pharmacological vs pharmacological or non-pharmacological vs any intervention) [12]. If a network included pharmacological interventions and a placebo or control, then it was classified as pharmacological vs placebo/control comparison type. Networks with pharmacological treatments but no placebo or control were categorised as a pharmacological vs pharmacological comparison type. Networks including at least one non-pharmacological treatment were classified as non-pharmacological vs any intervention comparison type. We classified NMAs according to the structure of the network (i.e. open networks vs networks with at least one closed loop of evidence) and the type of analyses presented (Bayesian, frequentist, or both). We also categorised NMAs according to their type of funding, irrespective of authors' funding, as industry-sponsored, publicly sponsored, mixed-funded, non-sponsored studies, and funding not reported.

We assessed the completeness of reporting for each NMA, including main manuscript and online supplements, using the PRISMA-NMA checklist, which includes 32 items in total: 27 core items as indicated in the core 2009 PRISMA Statement and five additional items (S1-S5) specific to the reporting of NMAs. We also prepared a modified version of the PRISMA-NMA checklist such that multiple items could be listed an evaluated separately at a more granular level, which resulted in 49 items (Appendix Table 1); this included for example two separate terms for systematic review and NMA/ related form of meta-analysis in the title, instead of a single item. We assigned each component a 'yes' (1 point) or 'no' (0 points) depending on whether it was reported. We ended up with a scale of 32 points for the original PRISMA-NMA items (termed 'original PRISMA-NMA score') and a scale of 49 points for the modified checklist ('modified PRISMA-NMA score').

\section{Statistical analysis}

We compared reporting scores (both original and modified PRISMA-NMA scores, as described in the data abstraction section) between NMAs published in the interval January 2013 to December 2015 and January 2016 to December 2018. We performed a descriptive analysis for the PRISMA-NMA items (reporting percentage per item) and presented the percentage of studies with adequate reporting for each item prior to and after the PRISMA-NMA publication. We evaluated whether there was a total improvement in reporting over publication year using the Cox and Stuart trend test (null hypothesis: there is not a monotonic trend) in the trend library in $\mathrm{R}$ [14].

We compared reporting scores between Cochrane NMAs, non-Cochrane NMAs with a protocol, and nonCochrane NMAs without a protocol. In non-Cochrane NMAs, we considered a protocol to be available only when this was reported in the manuscript (including registration in PROSPERO). Reporting scores were additionally compared between journals endorsing the original PRISMA and journals that did not recommend using PRISMA in their submission guidelines, as reported in http://www.prisma-statement.org/Endor sement/PRISMAEndorsers. We calculated the mean percent score difference between 2013 and 2015 and 2016 and 2018 along with a 95\% confidence interval (CI) per journal with impact factor $>10$. We also calculated the mean and median scores for each scale, along with the $95 \% \mathrm{CI}$ or interquartile range (IQR), respectively.

We conducted a univariable regression analysis assessing the overall impact of year of publication as a dichotomous variable until vs. after 2015 on the PRISMA-NMA score. We also performed two univariable regression analyses to assess the impact of year of publication on the PRISMA-NMA score, for studies published before and after 2015, separately. Similarly, we performed a univariable regression for studies published until and after the PRISMA-NMA publication focusing only on the NMA specific items S1-S5 (min score 0 , max score 5). To evaluate jointly the influence of the journal impact factor, year of publication, treatment type (pharmacological vs. non-pharmacological), funding type (industry or mixed vs. other), and review type (review with protocol vs. review without protocol) on reporting scores, we performed a multivariable regression analysis model. In case the impact factor was not available for a journal, we considered it as a zero value. We labelled the treatment type pharmacological when the network was categorised as pharmacological vs placebo/control or as pharmacological vs pharmacological comparison type, and the nonpharmacological treatment type when the network was categorised as non-pharmacological vs any. A network had an industry or mixed funding type when at least one of the sponsors for the review itself was industry. Each review was also classified depending on the protocol availability. We decided to use a binary categorisation of each covariate to improve power in our analysis. We also performed a multivariable regression using the same covariates apart from year of publication, which was considered as a dichotomous variable until vs. after 2015, as a subsequent sensitivity analysis. For our analyses, we used both original and modified PRISMA-NMA scores, 
a significance level of 5\%, and the $\mathrm{R}$ software version 3.6.2 (R Development Core Team 2019) [15].

\section{Results}

\section{Literature search}

The updated literature search yielded 4871 citations (Fig. 1). We included an additional of 489 citations published between 2013 and 2018 from supplementary sources and our published scoping reviews [7, 8, 12]. After de-duplication (i.e. multiple publications of the same systematic review and NMA), we screened 4446 unique titles and abstracts and 2212 full-text citations. Overall, we included 1144 NMAs that fulfilled the eligibility criteria (Appendix Table 2).

In the following, NMA reporting is presented according to the modified PRISMA-NMA score. Results according to the original PRISMA-NMA score are presented in the supplementary files.

\section{Study and method characteristics}

The number of NMAs published by year between 2013 and 2018 along with the per-year average score across years is shown in Fig. 2 (see also Appendix Fig. 1 for the original PRISMA-NMA score). The highest mean reporting score was observed in 2018, whereas the $66 \%$ of the NMAs (755 of 1144 NMAs) were published in 2016-2018. The majority of the corresponding authors had an affiliation with China (357, 31\%), the USA (184, $16 \%)$, and the UK (164, 14\%) (Table 1, Appendix Fig. 2). Of the 450 journals included in our database, only $33 \mathrm{had}$ already adopted the original PRISMA guidance. NMAs were published in journals with a median impact factor of 3.74 (IQR 2.69-5.81; Appendix Fig. 3). Among the included reviews, 801 (70\%) NMAs employed a Bayesian hierarchical approach alone $(779,68 \%)$ or in addition to a frequentist approach $(22,2 \%)$. Most networks included pharmacological treatments only or in addition to a placebo/control treatment (907, 79\%). We identified 33 Cochrane reviews with a protocol (3\% of the networks), 280 non-Cochrane reviews with a protocol $(24 \%$ of the networks), and 831 non-Cochrane reviews without a protocol (73\% of the networks).

Five in six networks included at least one closed loop of evidence $(958,84 \%)$. A total of 398 NMAs (35\%) were publicly funded, but funding was not reported in 314 (27\%) NMAs. The most popular journals in our NMA database were PLOS ONE $(n=52,5 \%)$ followed by Oncotarget $(43 ; 4 \%)$ and Medicine $(42 ; 4 \%)$.

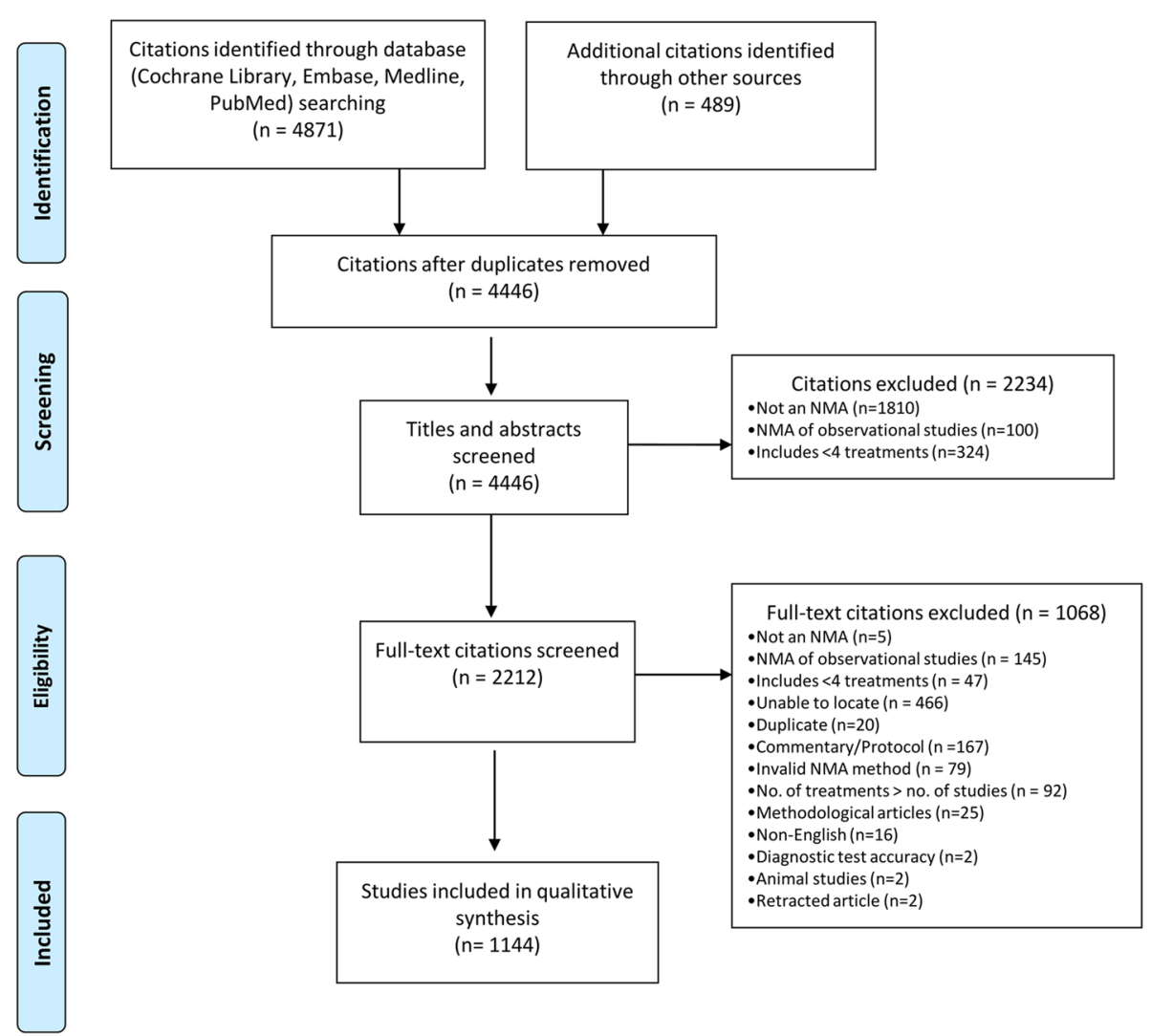

Fig. 1 Flow diagram of the selection process for the included systematic reviews and network meta-analyses 


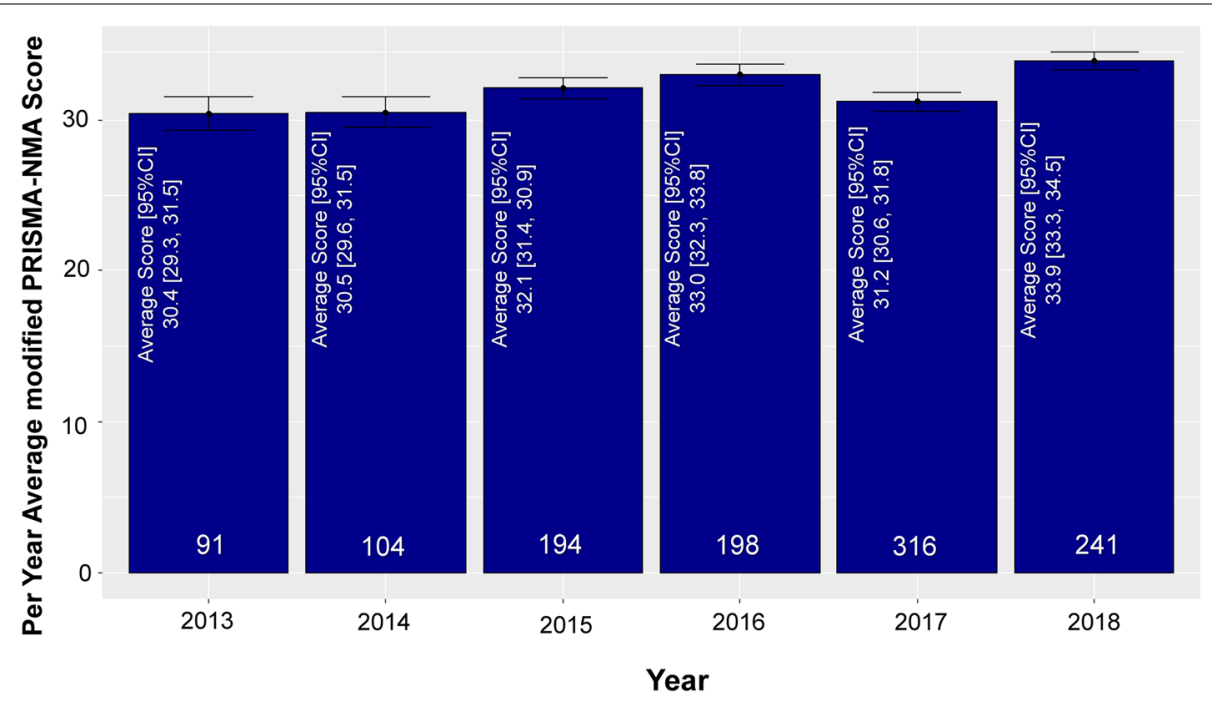

Fig. 2 Number of systematic reviews and network meta-analyses, and per-paper average modified PRISMA-NMA score published between 2013 and $2018^{* \prime}$ denotes that the search was performed up to July 2018, and thus only 7 months of that year are reflected in this graph. Error bars parallel to the $y$-axis represent the uncertainty of the modified PRISMA-NMA score

\section{Comprehensiveness of reporting in network meta-analyses Reporting score overall, until and after 2015}

The modified PRISMA-NMA of 49 items had a mean score 32.1 (95\% CI 31.8-32.4; Appendix Fig. 4a; Appendix Fig. 5a). The mean score of NMAs published until and after 2015 were 31.3 (95\% CI 30.8-31.8) and 32.6 (95\% CI 32.2-33.0) (Appendix Figs. 4b and 5b). Although reporting score increased per year across NMAs, the increase was small and not statistically significant (trend test $p$-value $=0.480$, Appendix Fig. 6).

Findings from univariable regression analysis showed that after 2015 reporting of NMAs improved by an average score of 1.25 (95\% CI 0.59 to 1.91; Table 2). Univariable regression analysis showed that the PRISMA-NMA score until 2015 is positively associated with year, and for 1 -year increase the score increases by 0.96 items (95\% CI 0.32 to 1.59). For NMAs published after 2015, the score increases by 0.53 (95\% CI 0.02 to 1.04) for 1-year increase. Focusing only on the NMA-specific items S1-S5, the speed of improvement was higher before the publication of the PRISMA-NMA guidelines (average per-year score increase in items S1-S5: 2013-2015 0.32 95\% CI 0.14 to 0.49 ; $2016-20180.2295 \%$ CI 0.11 to 0.33 ; Appendix Table 3).

\section{Factors that impact on reporting}

On average, articles published in journals endorsing the original PRISMA had higher modified score (mean 34.5, 95\% CI 33.8-35.2) compared with articles published in journals not explicitly endorsing PRISMA (median 31.7, IQR 21.4-32.0), yet this is not substantial (Fig. 3c;
Appendix Fig. 4c). Overall, reporting of NMAs has been improved within each journal after 2015 (Appendix Table 4). Reporting did not vary substantially across continents (Appendix Fig. 7(c)(d)). Reporting differed across the types of reviews, with Cochrane reviews being associated with the highest scores (Cochrane reviews: mean 36.8, 95\% CI 35.6-38.0; non-Cochrane reviews with protocol: mean 36.2, 95\% CI 35.7-36.7; non-Cochrane reviews without a protocol median 30.6, 95\% CI 29.830.4, Appendix Fig. 7(i)(j)).

Findings from multivariable regression analysis including year as a continuous variable showed that there was an increase in PRISMA score per year by 0.34 items (95\% CI increase 0.16 to 0.52 ) when adjusting for journal impact factor, type of review, funding, and treatment types being compared in the network. Reporting was analogous to the journal impact factor (average scoreincrease 0.10 items, 95\% CI increase 0.07 to 0.13 ). A positive association was also observed with publicly/nonsponsored NMAs (average score-increase 1.34 items, 95\% CI increase 0.56 to 2.11 ; mean score: 32.5 , $95 \%$ CI 32.2-32.8). Findings from multivariable regression analysis including year as a binary variable (before and after 2015) were in agreement with the multivariable regression and the year as a continuous variable, and suggested that there was an improvement in PRISMA score after 2015 by 0.81 items ( $95 \%$ CI increase 0.23 to 1.39 ) when adjusting for journal impact factor, type of review, funding, and treatment types being compared in the network. Conversely, a negative association was observed between the PRISMA-NMA score and reviews without a protocol 
Table 1 Characteristics of NMAs published between 2013 and 2018

\begin{tabular}{|c|c|c|c|c|c|c|c|c|c|}
\hline \multirow[t]{3}{*}{ Published in journals endorsing NMA } & \multicolumn{3}{|c|}{$\begin{array}{l}\text { NMAs published between } \\
2013 \text { and } 2015\end{array}$} & \multicolumn{3}{|c|}{$\begin{array}{l}\text { NMAs published between } \\
2016 \text { and } 2018\end{array}$} & \multicolumn{3}{|l|}{ Total } \\
\hline & Yes & No & Total & Yes & No & Total & Yes & No & Total \\
\hline & $88(23 \%)$ & $301(77 \%)$ & 389 & $82(11 \%)$ & $673(89 \%)$ & 755 & $170(15 \%)$ & $974(85 \%)$ & 1144 \\
\hline \multicolumn{10}{|c|}{ Ten most prevalent countries of corresponding author: frequency (\%) } \\
\hline China & $17(22 \%)$ & $60(78 \%)$ & 77 & $21(8 \%)$ & $256(92 \%)$ & 277 & $38(11 \%)$ & $316(89 \%)$ & 354 \\
\hline USA & $17(22 \%)$ & $61(78 \%)$ & 78 & $12(11 \%)$ & 94 (89\%) & 106 & $29(16 \%)$ & 155 (84\%) & 184 \\
\hline UK & $16(22 \%)$ & $58(78 \%)$ & 74 & $13(14 \%)$ & 77 (86\%) & 90 & $29(18 \%)$ & 135 (82\%) & 164 \\
\hline Canada & $10(29 \%)$ & $24(71 \%)$ & 34 & $8(20 \%)$ & $32(80 \%)$ & 40 & $18(24 \%)$ & $56(76 \%)$ & 74 \\
\hline Italy & $5(16 \%)$ & $26(84 \%)$ & 31 & $4(13 \%)$ & $28(88 \%)$ & 32 & $9(14 \%)$ & $54(86 \%)$ & 63 \\
\hline Korea (South) & $1(25 \%)$ & $3(75 \%)$ & 4 & $3(10 \%)$ & $28(90 \%)$ & 31 & $4(11 \%)$ & $31(89 \%)$ & 35 \\
\hline Germany & $3(20 \%)$ & $12(80 \%)$ & 15 & $1(6 \%)$ & $16(94 \%)$ & 17 & $4(13 \%)$ & $28(88 \%)$ & 32 \\
\hline France & $2(20 \%)$ & $8(80 \%)$ & 10 & $2(17 \%)$ & $10(83 \%)$ & 12 & $4(18 \%)$ & $18(82 \%)$ & 22 \\
\hline Switzerland & $4(40 \%)$ & $6(60 \%)$ & 10 & $3(30 \%)$ & $7(70 \%)$ & 10 & $7(35 \%)$ & $13(65 \%)$ & 20 \\
\hline Japan & $2(40 \%)$ & $3(60 \%)$ & 5 & $0(0 \%)$ & $15(100 \%)$ & 15 & $2(10 \%)$ & $18(90 \%)$ & 20 \\
\hline \multicolumn{10}{|l|}{ Ten most prevalent journals: frequency (\%) } \\
\hline Plos One & $31(100 \%)$ & NA & 31 & $21(100 \%)$ & NA & 21 & $52(100 \%)$ & NA & 52 \\
\hline Oncotarget & NA & $4(100 \%)$ & 4 & NA & $39(100 \%)$ & 39 & NA & $43(100 \%)$ & 43 \\
\hline Medicine & NA & $9(100 \%)$ & 9 & NA & $33(100 \%)$ & 33 & NA & $42(100 \%)$ & 42 \\
\hline Cochrane Database Of Systematic Reviews & NA & $16(100 \%)$ & 16 & NA & $17(100 \%)$ & 17 & NA & $33(100 \%)$ & 33 \\
\hline Scientific Reports & NA & $2(100 \%)$ & 2 & NA & $25(100 \%)$ & 25 & NA & $27(100 \%)$ & 27 \\
\hline Current Medical Research And Opinion & NA & $11(100 \%)$ & 11 & NA & $10(100 \%)$ & 10 & NA & $21(100 \%)$ & 21 \\
\hline $\mathrm{BMJ}$ & $16(100 \%)$ & NA & 16 & $5(100 \%)$ & NA & 5 & $21(100 \%)$ & NA & 21 \\
\hline Health Technology Assessment & NA & $8(100 \%)$ & 8 & NA & $11(100 \%)$ & 11 & NA & $19(100 \%)$ & 19 \\
\hline Alimentary Pharmacology \& Therapeutics & $8(100 \%)$ & NA & 8 & $10(100 \%)$ & NA & 10 & NA & $18(100 \%)$ & 18 \\
\hline Clinical Therapeutics & NA & $7(100 \%)$ & 7 & NA & $8(100 \%)$ & 8 & NA & $15(100 \%)$ & 15 \\
\hline \multicolumn{10}{|l|}{ Type of review: frequency (\%) } \\
\hline Non-Cochrane review without protocol & $70(23 \%)$ & $236(77 \%)$ & 306 & $42(8 \%)$ & $483(92 \%)$ & 525 & $112(13 \%)$ & $719(87 \%)$ & 831 \\
\hline Non-Cochrane review with protocol & $18(27 \%)$ & $49(73 \%)$ & 67 & $40(19 \%)$ & $173(81 \%)$ & 213 & $58(21 \%)$ & $222(79 \%)$ & 280 \\
\hline Cochrane review & $0(0 \%)$ & $16(100 \%)$ & 16 & $0(0 \%)$ & $17(100 \%)$ & 17 & $0(0 \%)$ & $33(100 \%)$ & 33 \\
\hline \multicolumn{10}{|l|}{ Type of treatment group: frequency (\%) } \\
\hline Pharmacological vs Placebo & $44(20 \%)$ & $171(80 \%)$ & 215 & $50(14 \%)$ & $315(86 \%)$ & 365 & $94(16 \%)$ & $486(84 \%)$ & 580 \\
\hline Pharmacological vs Pharmacological & $27(26 \%)$ & 77 (74\%) & 104 & $16(7 \%)$ & $207(93 \%)$ & 223 & $43(13 \%)$ & $284(87 \%)$ & 327 \\
\hline Non-pharmacological vs Any treatment & $17(24 \%)$ & $53(76 \%)$ & 70 & $16(10 \%)$ & $151(90 \%)$ & 167 & $33(14 \%)$ & $204(86 \%)$ & 237 \\
\hline \multicolumn{10}{|l|}{ Shape of network: frequency (\%) } \\
\hline Full shaped with at least one closed loop & $71(22 \%)$ & $245(78 \%)$ & 316 & $69(11 \%)$ & $573(89 \%)$ & 642 & $140(15 \%)$ & $818(85 \%)$ & 958 \\
\hline Open shaped with no closed loops & $17(24 \%)$ & $55(76 \%)$ & 72 & $13(12 \%)$ & $94(88 \%)$ & 107 & $30(17 \%)$ & $149(83 \%)$ & 179 \\
\hline Unclear & $0(0 \%)$ & $1(100 \%)$ & 1 & $0(0 \%)$ & $6(100 \%)$ & 6 & $0(0 \%)$ & $7(100 \%)$ & 7 \\
\hline \multicolumn{10}{|l|}{ Presentation results: frequency $(\%)^{\mathrm{a}}$} \\
\hline \multicolumn{10}{|l|}{ Presentation of NMA results } \\
\hline Ranking statistics & $46(25 \%)$ & $138(75 \%)$ & 363 & $58(11 \%)$ & $494(89 \%)$ & 552 & $104(14 \%)$ & $632(86 \%)$ & 736 \\
\hline Forest plot & $48(24 \%)$ & $149(76 \%)$ & 197 & $60(12 \%)$ & $449(88 \%)$ & 509 & $108(15 \%)$ & $598(85 \%)$ & 706 \\
\hline League tables & $37(23 \%)$ & $124(77 \%)$ & 161 & $48(10 \%)$ & $419(90 \%)$ & 467 & $85(14 \%)$ & $543(86 \%)$ & 628 \\
\hline \multicolumn{10}{|l|}{ Analysis setting: frequency (\%) } \\
\hline Bayesian & $67(24 \%)$ & $215(76 \%)$ & 272 & $56(11 \%)$ & $441(89 \%)$ & 497 & $123(16 \%)$ & $656(84 \%)$ & 779 \\
\hline Frequentist & $19(19 \%)$ & $79(81 \%)$ & 98 & $26(11 \%)$ & $209(89 \%)$ & 235 & $45(14 \%)$ & $288(86 \%)$ & 333 \\
\hline Both & $0(0 \%)$ & $1(100 \%)$ & 1 & $0(0 \%)$ & $21(100 \%)$ & 21 & $0(0 \%)$ & $22(100 \%)$ & 22 \\
\hline Unclear & $2(25 \%)$ & $6(75 \%)$ & 8 & $0(0 \%)$ & $2(100 \%)$ & 2 & $2(20 \%)$ & $8(80 \%)$ & 10 \\
\hline
\end{tabular}

Bayesian analysis settings: frequency (\%) ${ }^{\mathrm{a}}$ 
Table 1 (continued)

\begin{tabular}{|c|c|c|c|c|c|c|c|c|c|}
\hline \multirow[t]{3}{*}{ Published in journals endorsing NMA } & \multicolumn{3}{|c|}{$\begin{array}{l}\text { NMAs published between } \\
2013 \text { and } 2015\end{array}$} & \multicolumn{3}{|c|}{$\begin{array}{l}\text { NMAs published between } \\
2016 \text { and } 2018\end{array}$} & \multicolumn{3}{|l|}{ Total } \\
\hline & Yes & No & Total & Yes & No & Total & Yes & No & Total \\
\hline & $88(23 \%)$ & $301(77 \%)$ & 389 & $82(11 \%)$ & $673(89 \%)$ & 755 & $170(15 \%)$ & $974(85 \%)$ & 1144 \\
\hline Reported prior distributions & $37(24 \%)$ & $117(76 \%)$ & 154 & $22(12 \%)$ & $169(88 \%)$ & 191 & $59(17 \%)$ & $286(83 \%)$ & 345 \\
\hline Model fit assessment & $40(26 \%)$ & $112(74 \%)$ & 152 & $21(11 \%)$ & $169(89 \%)$ & 190 & $61(18 \%)$ & $281(82 \%)$ & 342 \\
\hline Used different priors as additional analyses & $5(28 \%)$ & $13(72 \%)$ & 18 & $4(24 \%)$ & $13(76 \%)$ & 17 & $9(26 \%)$ & $26(74 \%)$ & 35 \\
\hline \multicolumn{10}{|l|}{ Additional analyses: frequency $(\%)^{\mathrm{a}}$} \\
\hline \multicolumn{10}{|l|}{ Additional NMA analyses } \\
\hline Subgroup and/or sensitivity analysis & $50(28 \%)$ & $131(72 \%)$ & 181 & $46(15 \%)$ & $266(85 \%)$ & 312 & $96(19 \%)$ & $397(81 \%)$ & 493 \\
\hline Meta-regression & $18(26 \%)$ & $52(74 \%)$ & 70 & $18(17 \%)$ & $87(83 \%)$ & 105 & $36(21 \%)$ & $139(79 \%)$ & 175 \\
\hline Alternative treatment formulations in the network & $13(42 \%)$ & $18(58 \%)$ & 31 & $3(10 \%)$ & $26(90 \%)$ & 29 & $16(27 \%)$ & $44(73 \%)$ & 60 \\
\hline \multicolumn{10}{|c|}{ Funding: frequency (\%) } \\
\hline Publicly sponsored & $27(21 \%)$ & $104(79 \%)$ & 131 & $29(11 \%)$ & $238(89 \%)$ & 267 & $56(14 \%)$ & $342(86 \%)$ & 398 \\
\hline Funding source not reported & $8(9 \%)$ & $82(91 \%)$ & 90 & $14(6 \%)$ & $210(94 \%)$ & 224 & $22(7 \%)$ & $292(93 \%)$ & 314 \\
\hline Non-sponsored & $34(37 \%)$ & $59(63 \%)$ & 93 & $26(15 \%)$ & $143(85 \%)$ & 169 & $60(23 \%)$ & $202(77 \%)$ & 262 \\
\hline Industry-sponsored & $16(24 \%)$ & $52(76 \%)$ & 68 & $11(13 \%)$ & $72(87 \%)$ & 83 & $27(18 \%)$ & $124(82 \%)$ & 151 \\
\hline Mixed-funding & $3(43 \%)$ & $4(57 \%)$ & 7 & $2(17 \%)$ & $10(83 \%)$ & 12 & $5(26 \%)$ & $14(74 \%)$ & 19 \\
\hline
\end{tabular}

${ }^{a}$ The total number of NMAs does not add up to 1144 as each article might pertain to more than one category

NMA network meta-analysis

(average score-decrease 5.18 items, 95\% CI decrease 4.55 to 5.80 ; mean score $30.6,95 \%$ CI $30.2-30.9$ ), and networks including pharmacological treatments (average score-decrease 0.71 items $95 \%$ CI decrease 0.04 to 1.40 ; mean score 31.8, 95\% CI 31.4-32.1; Table 2; Appendix Table 3).

\section{PRISMA-NMA items that improved and items that still need attention}

The percentage of adequately reported items before and after the PRISMA-NMA statement publication is presented in Fig. 3. Reporting was improved in 23 (47\%) items, including the five items (S1-S5) specific to the reporting of the synthesis in NMAs. Overall, of the 958 with at least one closed loop, 693 networks (72\%) reported consistency investigation, and this has improved over the years (range 52-78\% NMAs). After 2015 , improvement was also observed in items about the overview of the network and study characteristics.

However, reporting of several other items has shown little or no improvement after 2015, while adequate reporting of some items has declined, signalling a need for further attention. Description of summary effect sizes to be used, presentation of individual study data, sources of funding for the systematic review, and role of funders dropped in frequency after 2015 by $6-16 \%$ (Fig. 3). In particular, the role of funders for the systematic review was consistently missing across years for 71-88\% NMAs (Appendix Table 5). A total of 294 NMAs (26\%) reported the existence of a protocol, of which 229 (78\%) reported a registry or a web site where a protocol could be accessed, and $79(34 \%)$ of these NMAs reported this information in the abstract as well. Details on methods to assess bias across studies and on conducting additional analyses were underreported across all years.

\section{Discussion}

Our findings suggest that key reporting items are missing in the majority of published NMAs. While minor improvements in the reporting of some elements were noted following PRISMA-NMA, other elements also experienced reductions. In total, reporting has improved after 2015 in 47\% of the modified PRISMA-NMA items, but it has also deteriorated in $49 \%$ of the core items. Improvement was observed in items about the NMA synthesis, overview of the network and study characteristics, while deterioration was noticed in the description of summary effect sizes to be used, presentation of individual study data, sources of funding for the systematic review, and role of funders.

An explanation of the decrease in inadequately reported items may be restriction in the word count required by most journals. Also, some of the required details may be reported in the study's protocol. Another key item that was inadequately reported was funding status. Journal guidelines highlight funding as crucial information to be reported in a paper; however, we noticed that mainly the author funding is reported and 
Table 2 Univariable and multivariable regression using the modified PRISMA-NMA

\begin{tabular}{|c|c|c|c|}
\hline Covariates & Interpretation of the coefficient & Coefficient $(95 \% \mathrm{Cl})$ & Sample size \\
\hline \multicolumn{4}{|l|}{ Univariable analyses and subgroups } \\
\hline Published after 2015 vs until 2015 & Average increase in the score after 2015 & $1.25(0.59,1.91)$ & \multirow{3}{*}{$\begin{array}{l}\text { Before 2015: } 389 \\
\text { After 2015: } 755\end{array}$} \\
\hline $\begin{array}{l}\text { Year of publication, subgroup: } \\
\text { only NMAs published before } 2015\end{array}$ & Average increase in the score per year & $0.96(0.32,1.59)$ & \\
\hline $\begin{array}{l}\text { Year of publication, subgroup: } \\
\text { only NMAs published after } 2015\end{array}$ & Average increase in the score per year & $0.53(0.02,1.04)$ & \\
\hline \multicolumn{4}{|c|}{ Multivariable analyses with year as a continuous variable } \\
\hline Year of publication & Average increase in the score per year & $0.34(0.16,0.52)$ & $\begin{array}{l}\text { Year 2013: } 91 \text { (reference group) } \\
\text { Year 2014: } 104 \\
\text { Year 2015: } 194 \\
\text { Year 2016: } 198 \\
\text { Year 2017: } 316 \\
\text { Year 2018: } 241\end{array}$ \\
\hline Treatment type & $\begin{array}{l}\text { Average increase in the score if network } \\
\text { includes pharmacological treatments }\end{array}$ & $-0.66(-1.34,0.02)$ & $\begin{array}{l}\text { Pharmacological treatments: } 907 \\
\text { Non-pharmacological treatments (refer- } \\
\text { ence group): } 237\end{array}$ \\
\hline Funding type & $\begin{array}{l}\text { Average increase in the score if non- } \\
\text { sponsored/publicly sponsored }\end{array}$ & $1.34(0.56,2.11)$ & $\begin{array}{l}\text { Non-sponsored/publicly sponsored/not } \\
\text { reported: } 974 \\
\text { Industry/mixed sponsored (reference } \\
\text { group): } 170\end{array}$ \\
\hline Review type & $\begin{array}{l}\text { Average increase in the score if protocol } \\
\text { is not available/reported }\end{array}$ & $-5.12(-5.74,-4.49)$ & $\begin{array}{l}\text { With protocol (reference group): } 313 \\
\text { Without protocol: } 831\end{array}$ \\
\hline Impact factor & $\begin{array}{l}\text { Average increase in the score per impact } \\
\text { factor increase ( } 1 \text { unit) }\end{array}$ & $0.10(0.07,0.13)$ & \\
\hline \multicolumn{4}{|c|}{ Multivariable analyses with year as a dichotomous variable } \\
\hline Year of publication & Average increase in the score per year & $0.81(0.23,1.39)$ & $\begin{array}{l}\text { Before } 2015 \text { (reference group):389 } \\
\text { After 2015:755 }\end{array}$ \\
\hline Treatment type & $\begin{array}{l}\text { Average increase in the score if network } \\
\text { includes pharmacological treatments }\end{array}$ & $-0.72(-1.40,-0.04)$ & $\begin{array}{l}\text { Pharmacological treatments: } 907 \\
\text { Non-pharmacological treatments (refer- } \\
\text { ence group): } 237\end{array}$ \\
\hline Funding type & $\begin{array}{l}\text { Average increase in the score if non- } \\
\text { sponsored/publicly sponsored }\end{array}$ & $1.36(0.58,2.14)$ & $\begin{array}{l}\text { Non-sponsored/publicly sponsored/Not } \\
\text { reported: } 974 \\
\text { Industry/mixed sponsored (reference } \\
\text { group): } 170\end{array}$ \\
\hline Review type & $\begin{array}{l}\text { Average increase in the score if protocol } \\
\text { is not available/reported }\end{array}$ & $-5.18(-5.8,-4.55)$ & $\begin{array}{l}\text { With protocol (reference group): } 313 \\
\text { Without protocol: } 831\end{array}$ \\
\hline Impact factor & $\begin{array}{l}\text { Average increase in the score per impact } \\
\text { factor increase (1 unit) }\end{array}$ & $0.10(0.06,0.13)$ & \\
\hline
\end{tabular}

$\mathrm{Cl}$ confidence interval

not funding for the review itself. Funding for the systematic review and role of funders are consistently underreported, which is a cause of concern. Presentation of individual study data is another item that has worsened after 2015. This may be because larger and more complex networks are being structured compared to past years or because of authors' desire to retain ownership of the data, given the large efforts to compile the data sets, and to potentially publish new work after additional evidence (e.g. treatments) emerge.

Key factors that may impact the reporting were the journal's impact factor, funding type, year of publication, type of review, and treatment category included in the network. In particular, newer and publicly sponsored NMAs of non-pharmacological therapies with a protocol, and published in high impact factor journals, were associated with better reporting. Our results showed that reviews with a protocol, and particularly Cochrane reviews, were associated with higher PRISMA-NMA scores.

Overall, reporting is adequate but not high (mean PRISMA-NMA score 32.1; 95\% CI 31.8-32.4; $\max$ 49). Authors of NMAs showed a steep improvement in earlier years (2013-2015), but it stabilises after the PRISMA-NMA guideline publication. The improvement continues to exist throughout the years, but the 


\begin{tabular}{|c|c|c|c|}
\hline \multirow{2}{*}{ PRISMANMA item } & \multicolumn{3}{|c|}{ Mbdfied PRISMA-NMA score } \\
\hline & $2013-2015 \%)$ & $2016-2018 \%$ & Overall (\%) \\
\hline DIS CUSSION: Sunmary ofkey fnding, inchoding strength of evidente & 99 & 97 & 98 \\
\hline ABS TRACT: min objectives & 97 & 95 & 96 \\
\hline ABS TRACT: conchusions and inplications of findings & 96 & 95 & 96 \\
\hline INTRODUCTON: Rationala for systematic reviaw and network met-amalysis & 96 & 93 & 94 \\
\hline 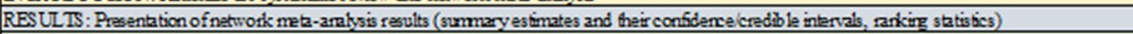 & 96 & 95 & 96 \\
\hline METHODS: Descripbion of analysis methods (eg, network meta-analysis method) & 95 & 93 & 94 \\
\hline METHODS: Description of irformz tion sources with searchdates & 95 & 95 & 95 \\
\hline ABS TRACT: synthesis methods ( $\mathrm{eg}$, nettrock meta-znalysis) & 94 & 92 & 93 \\
\hline METHODS: Description of summarymeasures to be used (e.s. odds ratio, mezn difference) & 94 & 88 & 90 \\
\hline DIS CUSS ION: General interpretation of results, comparison to othr evidenre, and inplications for fubre research & 93 & 92 & 92 \\
\hline DIS CUSSION: Discussion of shudy limitations & 92 & 94 & 93 \\
\hline RESULTS: Nurber of studies screened and inchded in the review, and reasors for exchsion (e.c. flow diagram) & 92 & 91 & 91 \\
\hline METHODS: Rationale for eligibilitycriteria (eg. PICO criteria length of follow-up, vears considered, language, publication status) & 91 & 93 & 92 \\
\hline INTRODUCTION: Evplicit stetement of the Questions beng addressed (PICO criteria) & 88 & 85 & 86 \\
\hline RESULTS: Presentation of characteristios per study y w th citations (ex, ina table) & 88 & 91 & 90 \\
\hline ABS TRACT: PICO criteria & 85 & 7 & 80 \\
\hline ABS TRACT: Stuctured summary & 85 & 78 & 80 \\
\hline ABS TRACT: Number of studies and parbicipants & 83 & 83 & 83 \\
\hline METHODS: Description of the study selection process & 82 & 83 & 83 \\
\hline IIIIE: Netro ork Meta-analysis or related fom of meth-analysis & 82 & 89 & 87 \\
\hline RESULTS: Brif ovemiens of nensoik characteristics (S4) & 80 & 84 & 83 \\
\hline 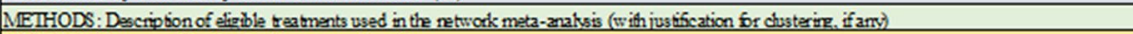 & 78 & 84 & 82 \\
\hline FUNDNG: Sources of finding for the systematic reviaw & 76 & 68 & 71 \\
\hline METHODS: Description of methods used to assess stud y risk of bias & 76 & 80 & 79 \\
\hline RESULTS: Nensark plos (S3) & 76 & 88 & 84 \\
\hline METHODS: Description of collected items & 66 & $\pi$ & 73 \\
\hline ABS TRACT: Surmary estimates and their corbidenos/ored ble intervals & 65 & 58 & 60 \\
\hline RESULTS: Presentation of nisk of bias per studv & 62 & 64 & 63 \\
\hline METHODS: Description of tha data collection process (method) & 61 & $\pi$ & 72 \\
\hline METHODS: Decrivtion of method teed to assess inconsistency (S2) & 60 & 69 & 66 \\
\hline METHODS: Description of ad ditional analkses (e.... sersitivity analysis) & 58 & 48 & 52 \\
\hline ABS TRACT: Data sources & 57 & 56 & 56 \\
\hline RESULTS: Presentation of individual study data & 55 & 39 & 44 \\
\hline RESULTS : Prsentation of fresvils of additional analsses (e $q$, sensitivity analysis) & 53 & 49 & 50 \\
\hline RESULTS: Decription of restitss from im estigation of inconzistency (SS) & 50 & 60 & 57 \\
\hline METHODS: Afullelactronic saarch strategy avaiable & 45 & 50 & 48 \\
\hline METHODS: Description of treatment ranlings to be wed (e.g, surface under the cumlative ranking curve, P-scores) & 42 & 71 & 61 \\
\hline IIILE: Sustemabc review & 36 & 38 & 37 \\
\hline 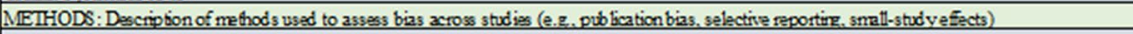 & 32 & 37 & 36 \\
\hline RESULTS: Presentation of results of bias asseasment across studies (e.?. finnel plot) & 28 & 34 & 32 \\
\hline FUNDING: Role of finders for the systematic revizi & 26 & 15 & 19 \\
\hline ABS TRACT: Intervention ankim (eg. surface under the cumvlative ranking cunve. P-scores) & 24 & 44 & 37 \\
\hline METHODS: Bistence of reviaw protocol 1 & 19 & 28 & 25 \\
\hline 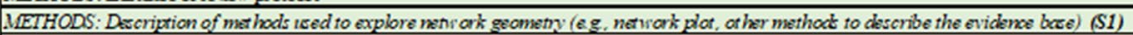 & 16 & 25 & 22 \\
\hline ABS TRACT: Limitations & 13 & 10 & 11 \\
\hline ABS TRACT: S wody zppraisal (e, , risk ofbizs) & 13 & 11 & 11 \\
\hline METHODS: Protocol can be accessed (e.E. Web address or re ristation nunber avalable) & 13 & 23 & 20 \\
\hline ABS IRACT: Funding & 9 & 5 & 6 \\
\hline ABS TRACT: Rezistation number withreqistrnname (e.z. PROSPERO) & 5 & 10 & 8 \\
\hline
\end{tabular}

Fig. 3 Plot of the percentage of adequately reporting the 49 modified PRISMA-NMA items overall and according to publication interval 2013-2015 and 2016-2018. PRISMA items are ordered from least to most well reported irrespective publication year. Statistically significant differences are indicated with a bold font. Each cell is coloured according to the reporting using the transformation of three colours: red (0\%), yellow (50\%), and green (100\%)

speed of improvement is lower between 2016 and 2018. This suggests that overall, the PRISMA-NMA guidance has not importantly affected reporting in new NMAs compared to older NMAs. This may be because NMA authors in the earlier years 2013-2015 already followed existing guidelines for standards of conduct of NMA through the International Society for Pharmacoeconomics and Outcomes Research (ISPOR) tools $[16,17]$ and National Institute for Clinical Excellence (NICE) Decision Support Unit's Evidence Synthesis Technical Support Documents (TSDs) documents [18]. However, we observed improvement in the five items (S1-S5) specific to the reporting of the synthesis section of an NMA after 2015, ranging between 4 and
$12 \%$. The improvement observed in reporting might be attributed at least in part to PRISMA-NMA, but may also be due to additional factors, such as the increase in registering or publishing of peer-reviewed protocols; protocol existence in NMAs has increased from 15-39\% between 2013 and 2018. Our analysis showed that there is a slight improvement in reporting in the year 2016 compared to the year 2017 (2016: mean modified PRISMA-NMA score 33.0, 95\% CI 32.3-33.8; 2017: mean modified PRISMA-NMA score 31.2, 95\% CI 30.6-31.8). This may be due to the impact factor of the journal that NMAs were published in. The median impact factor of the journals the NMAs were published 
in 2016 was 3.87 (IQR 2.49-5.56), whereas in 2017 was 3.50 (IQR 2.63-5.16) (Appendix Fig. 8).

In our database of NMAs, the PRISMA-NMA guideline is only endorsed by $7 \%$ of the journals in which the NMAs were published. This highlights the need for journals publishing systematic reviews and NMAs to adopt the PRISMA-NMA guidelines to improve reporting, and to request the checklist upon a manuscript submission. Based on our findings, we provide recommendations to update the PRISMA-NMA statement to facilitate its use by systematic reviewers, journal editors, and peer reviewers (see the section "Conclusions and recommendations for practice").

To the best of our knowledge, this is the largest review assessing the PRISMA extension guideline for NMA in more than 1000 systematic reviews and NMAs. Our findings are aligned with previous findings by Hutton et al. [11], who evaluated 89 NMAs of non-pharmacological therapies; Tonin et al. [10] who assessed 477 NMAs of pharmacological treatments; and Lee and Shin [9] who assessed 21 NMAs in dental care. In agreement with assessments in systematic reviews and meta-analyses, reporting in the 27 core PRISMA items was suboptimal [2]. A previous assessment on reporting of pairwise systematic reviews and meta-analyses on nursing interventions in patients with Alzheimer's disease before and after PRISMA publication showed an improvement in the average core PRISMA items from 17.11 to 20.83 score [19]. Our findings about PRISMA-NMA are not limited to a specific disease area and showed that the average core PRISMA items score did not importantly change before (19.58 items) and after 2015 (19.88 items).

In addition to assessing the PRISMA-NMA items in the included systematic reviews, we explored factors that play a key role in reporting of NMA. In agreement with Zarin et al. [7], we found that the prerequisite assumptions are not always considered; 28\% of NMAs (265 of 958 NMAs with a closed loop) did not report an assessment for consistency in their methods. However, similar to Petropoulou et al.s [8] findings, reporting improved a bit over the years.

A limitation of our study is that in our assessment we considered that a PRISMA component was reported only if relevant information was present in the underlying section of the manuscript, as indicated in the PRISMANMA guideline. Also, protocols were not assessed for reporting relevant details, since the PRISMA-NMA guideline refers only to the final manuscript for NMAs. In our study, we have not explored differences in NMAs pointing authors to the relevant protocols for methods details and the remaining NMAs. However, in our assessment, we considered all available supplementary files and appendices. Although we may have missed some details reported in the protocol, we expect that this could not importantly impact our results. Also, an important unmeasured confounder in reporting may have been journals with no word count restrictions, but we have not assessed this further. Another potential limitation is that our literature search was conducted up to July 2018, and we may have missed recently published NMAs that were reported well. Also, the impact of the PRISMA-NMA guideline may not immediately be seen in the reporting of published NMAs and may take more time to start using it. However, this is the largest NMA database that assessed reporting, and we expect that no major differences would be seen in our results regarding the overall trend in reporting. A risk of confounding may be associated with our results between industry-sponsored studies and pharmacological treatments. We found that both factors were associated with a decrease in reporting (of the 170 industry-sponsored NMAs, 162 [95\%] included pharmacological interventions only [with or without a placebo] in the network). We used a binary system (presence/absence) for the PRISMA-NMA items, but this may not be the best approach to assess adequacy of reporting. For example, authors may report that transitivity was assessed but without providing more details on this.

\section{Conclusions and recommendations for practice}

NMAs published after 2015 more frequently reported the five items associated with NMA (i.e. description of methods to explore network geometry, description of methods to assess inconsistency, network plot presentation, brief overview of network characteristics, description of results from investigations of inconsistency). However, several important items are underreported and the yearly improvement in reporting is small.

In conducting this research, we chose to split certain PRISMA-NMA items into more specific items for evaluation, moving from 32 to 49 items. This allowed us to highlight crucial aspects of NMA that were or were not reported across the years. To this end, we suggest that the PRISMA-NMA checklist be updated using the 49 items instead of the initially suggested 32 items. This will provide more in-depth guidance to review authors, reviewers, editors, and readers for adequate reporting in NMA. The 49 items are listed in Appendix Table 1. Clarifying the information presented in the PRISMANMA 32 items into 49 different items may increase word count, but will enhance transparency of reporting. Online appendix files can also be used for additional and supporting information of the systematic review and NMA.

The original or modified PRISMA-NMA guidelines should be used extensively by review authors and be adopted by a wider range of journals. Journals editors, 
peer-reviewers, and systematic review authors should use the PRISMA-NMA list on a regular basis to write evaluate and publish results from NMA, paying special attention to items that are still underreported as highlighted in Fig. 3.

\section{Supplementary Information}

The online version contains supplementary material available at https://doi. org/10.1186/s13643-021-01780-9.

Additional file 1: Appendix 1. Eligibility criteria, screening, study selection, and data abstraction. Appendix Tables. Appendix Figures.

Additional file 2: References of included studies.

\section{Acknowledgements}

We thank Alissa Epworth for conducting the initial literature search and Patricia Rios for helping organise the identified articles in the Synthesi.SR program (http://www.breakthroughkt.ca/login.php).

\section{Authors' contributions}

AAV conceptualised the study, designed and coordinated the study, screened literature for inclusion, participated in all the calibration exercises of data collection and reporting assessment, helped resolve data discrepancies, analysed the data, interpreted the findings, and wrote the manuscript. ST screened the literature search results; abstracted, coded, cleaned, and analysed the data; interpreted the findings; performed reporting assessment; and helped to write the draft manuscript. SZ screened the literature search results; abstracted, coded, and cleaned the data; performed reporting assessment; and formatted the manuscript. IP, KMK, PA, NP, and $\mathrm{CL}$ helped to screen the literature and/or abstracted data and/or performed reporting assessment. AN, TP, AC, SES, BH, ACT, and DM helped conceptualise the study. GS conceptualised and designed the study and helped to write the draft manuscript. All authors read, provided substantial edits and/or comments on the manuscript, and approved the final version of the manuscript that was submitted for publication.

\section{Funding}

This work was supported by the Swiss National Science Foundation grant/ award number 179158. AAV, ST, SZ, KMK, and DM were funded from the European Union's Horizon 2020 [No. 754936]. AN, TP, and GS have been supported by SNSF grant agreement 320030_179158. SES is funded by a Tier 1 Canada Research Chair in Knowledge Translation. ACT is funded by a Tier 2 Canada Research Chair in Knowledge Synthesis.

\section{Availability of data and materials}

The datasets used and/or analysed during the current study are available from the corresponding author on reasonable request.

\section{Declarations}

\section{Ethics approval and consent to participate}

Not applicable.

\section{Consent for publication}

Not applicable.

\section{Competing interests}

The authors declare that they have no competing interests. Dr Areti Angeliki Veroniki is a Senior Editor for the Systematic Reviews journal, but had no involvement with the peer review process or decision for publication. Coauthors of this paper who authored included papers were not involved in abstracting or appraising these studies. All other authors have no potential conflict of interest to declare.

\section{Author details}

'Department of Primary Education, School of Education, University of loannina, loannina, Greece. ${ }^{2}$ Knowledge Translation Program, Li Ka Shing Knowledge Institute, St. Michael's Hospital, Toronto, ON, Canada. ${ }^{3}$ Department of Hygiene, Social-Preventive Medicine and Medical Statistics, Medical School, Aristotle University of Thessaloniki, Thessaloniki, Greece. ${ }^{4}$ Department of Biostatistics, Faculty of Veterinary Medicine, Mustafa Kemal University, Tayfur Sökmen Kampüsü 31060, Antakya, Hatay, Turkey. ${ }^{5}$ Department of Orthodontics and Dentofacial Orthopedics, Dental School/Medical Faculty, University of Bern, Bern, Switzerland. ${ }^{6}$ Cochrane Hypertension Review Group and the Therapeutics Initiative, University of British Columbia, Vancouver, Canada. Institute of Medical Biometry and Statistics, Faculty of Medicine and Medical Center, University of Freiburg, Freiburg, Germany. ${ }^{8}$ Institute of Social and Preventive Medicine, University of Bern, Bern, Switzerland. ' Université de Paris, Research Center of Epidemiology and Statistics Sorbonne Paris Cité (CRESS UMR1 153), INSERM, INRA, Paris, France. ${ }^{10}$ Cochrane France, Paris, France. ${ }^{11}$ Department of Geriatric Medicine, University of Toronto, Toronto, ON, Canada. ${ }^{12}$ Ottawa Hospital Research Institute, Ottawa, ON, Canada. ${ }^{13}$ University of Ottawa School of Epidemiology and Public Health, Ottawa, ON, Canada. ${ }^{14}$ Epidemiology Division, Dalla Lana School of Public Health, University of Toronto, Toronto, ON, Canada. ${ }^{15}$ Paris Descartes University, Sorbonne Paris CitéFaculté de Médecine, Paris, France.

Received: 27 January 2021 Accepted: 28 July 2021

Published: 10 September 2021

\section{References}

1. Moher D, Liberati A, Tetzlaff J, Altman DG, Group P. Preferred reporting items for systematic reviews and meta-analyses: the PRISMA statement. PLOS Med. 2009;6(7):e1000097.

2. Page MJ, Moher D. Evaluations of the uptake and impact of the Preferred Reporting Items for Systematic reviews and Meta-Analyses (PRISMA) Statement and extensions: a scoping review. Syst Rev. 2017;6(1):263.

3. Page M J, McKenzie J E, Bossuyt P M, Boutron I, Hoffmann T C, Mulrow C D et al. The PRISMA 2020 statement: an updated guideline for reporting systematic reviews. BMJ. 2021;372:n71. https://doi.org/10.1136/bmj.n71.

4. Page MJ, Altman DG, Shamseer L, McKenzie JE, Ahmadzai N, Wolfe $D$, et al. Reproducible research practices are underused in systematic reviews of biomedical interventions. J Clin Epidemiol. 2018;94:8-18.

5. Carroll K, Hemmings R. On the need for increased rigour and care in the conduct and interpretation of network meta-analyses in drug development. Pharm Stat. 2016;15(2):135-42.

6. Hutton B, Salanti G, Caldwell DM, Chaimani A, Schmid CH, Cameron C, et al. The PRISMA extension statement for reporting of systematic reviews incorporating network meta-analyses of health care interventions: checklist and explanations. Ann Intern Med. 2015;162(11):777-84.

7. Zarin W, Veroniki AA, Nincic V, Vafaei A, Reynen E, Motiwala SS, et al. Characteristics and knowledge synthesis approach for 456 network meta-analyses: a scoping review. BMC Med. 2017;15(1):3.

8. Petropoulou M, Nikolakopoulou A, Veroniki AA, Rios P, Vafaei A, Zarin W, et al. Bibliographic study showed improving statistical methodology of network meta-analyses published between 1999 and 2015. J Clin Epidemiol. 2017:82:20-8

9. Lee DW, Shin IS. Critical quality evaluation of network meta-analyses in dental care. J Dent. 2018:75:7-11.

10. Tonin FS, Rotta I, Mendes AM, Pontarolo R. Network meta-analysis: a technique to gather evidence from direct and indirect comparisons. Pharmacy Practice. 2017;15(1):943.

11. Pratt M, Wieland S, Ahmadzai N, Butler C, Wolfe D, Pussagoda K, et al. A scoping review of network meta-analyses assessing the efficacy and safety of complementary and alternative medicine interventions. Syst Rev. 2020;9(1):97.

12. Nikolakopoulou A, Chaimani A, Veroniki AA, Vasiliadis $\mathrm{HS}$, Schmid $\mathrm{CH}$, Salanti G. Characteristics of networks of interventions: a description of a database of 186 published networks. PLoS ONE. 2014;9(1):e86754.

13. Papakonstantinou T. nmadata: $R$ package for accessing redcap database of network meta-analyses hosted by ISPM University of Bern. Available at https://github.com/esm-ispm-unibe-ch/nmadata. 
14. R Development Core Team. R: A language and environment for statistical computing. Vienna: R Foundation for Statistical Computing; 2008. ISBN 3-900051-07-0 https://www.R-project.org.

15. R Core Team. R: a language and environment for statistical computing. Vienna: R Foundation for Statistical Computing; 2019

16. Hoaglin DC, Hawkins N, Jansen JP, Scott DA, Itzler R, Cappelleri JC, et al. Conducting indirect-treatment-comparison and network-meta-analysis studies: report of the ISPOR Task Force on Indirect Treatment Comparisons Good Research Practices: part 2. Value Health J Int Soc Pharmacoecon Outcomes Res. 2011;14(4):429-37.

17. Jansen JP, Fleurence R, Devine B, Itzler R, Barrett A, Hawkins N, et al. Interpreting indirect treatment comparisons and network meta-analysis for health-care decision making: report of the ISPOR Task Force on Indirect Treatment Comparisons Good Research Practices: part 1. Value Health J Int Soc Pharmacoecon Outcomes Res. 2011;14(4):417-28.

18. Dias S, Welton NJ, Sutton AJ, Ades AE. NICE DSU Technical Support Document 2: A generalised linear modelling framework for pairwise and network meta-analysis of randomised controlled trials. 2011; last updated April 2014; Available from http://www.nicedsu.org.uk.

19. Sun X, Zhou X, Yu Y, Liu H. Exploring reporting quality of systematic reviews and Meta-analyses on nursing interventions in patients with Alzheimer's disease before and after PRISMA introduction. BMC Med Res Methodol. 2018;18(1):154

\section{Publisher's Note}

Springer Nature remains neutral with regard to jurisdictional claims in published maps and institutional affiliations.

Ready to submit your research? Choose BMC and benefit from:

- fast, convenient online submission

- thorough peer review by experienced researchers in your field

- rapid publication on acceptance

- support for research data, including large and complex data types

- gold Open Access which fosters wider collaboration and increased citations

- maximum visibility for your research: over $100 \mathrm{M}$ website views per year

At BMC, research is always in progress.

Learn more biomedcentral.com/submissions 